\title{
Guest Editorial Enabling Architectures and Technologies for Smart Cities
}

The vision of smart cities has became a reality. Technological advances in low power devices and reliable communication and overall system architectures made it happening. However, there are plenty of improvements that still can be employed to further increase the efficiency of relevent systems or to provide new approaches that improve exisiting solutions.

This special issue on Enabling Architectures and Technologies for Smart Cities of the JOURNAL OF COMMUNICATIONS SOFTWARE AND SYSTEMS aims to report on the recent advancements and developments in various aspects related to emerging hardware and software technologies enabling the IoT, such as RFID, WSN, system software architecture, integrated solutions, embedded systems, and so on.

This issue recommended totally 10 papers for publication based on the standard reviewing process, where at least two constructive reviews and with guest editors comment have been received. Papers are split in two main groups focused mainly on system architectures that improve reliability in smart cities ([1-5]), and hardware solutions ([6-10]) that are presented as either upgrades to exisiting solutions or new more efficient proposals. All of these were validated by analytics, simulations and/or testbed approaches.

In the work "An IoT-aware Architecture to improve Safety in Sports Environments" [1], the authors L. Catarinucci, D. D. Donno, L. Mainetti, L. Patrono, M.L. Stefanizzi, L. Tarricone propose novel architecture for automatic monitoring in IoT sport-based systems. The system collects environmental conditions and player physiological parameters through a hybrid sensing network, achieved by combining RFID, WSN, 6LoWPAN, and CoAP, while validated successfully through use-case scenarios.

In the work "Crowd-sensing our Smart Cities: a Platform for Noise Monitoring and Acoustic Urban Planning", [2] the authors M. Zappatore, A. Longo, M. Bochicchio present novel platform, named "City Soundscape", that deals with noise pollution in cities.

Editorial approved May 31, 2017 by Dinko Begušić. Date of publication: June 1, 2017.

P. Šlić and T. Perković are with the University of Split, Croatia. (e-mails: \{Petar.Solic, Toni.Perkovic\}@fesb.hr)

L. Patrono is with the University of Salento, Lecce, Italy. (e-mail: luigi.patrono@unisalento.it)

L. Atzori is with the University of Cagliari, Italy. (e-mail: 1.atzori@diee.unica.it)

Digital Object Identifier (DOI): 10.24138/jcomss.v13i2.371
The platform is given as Andorid-based mobile application that is able to collect acoustic measurements through embedded microphone of a smartphone.

This platform also offers a web-based application for city managers to monitor expected noise reductions, estimated buying and installation cost, expected range of effectiveness. Tests were conducted in the province of Brindisi (Southern Italy), where students have participated in educational activities centered on acoustics by performing several outdoor measurement campaigns.

In the work "An IoT-aware AAL System to Capture Behavioral Changes of Elderly People", [3] the authors L. Mainetti, L. Patrono, A. Secco, I. Sergi present the ongoing activities within the City4Age project, which is focused on the use of IoT technologies to develop an innovative AAL system able to capture personal data of elderly people in their home and city environments. An architecture is presented that exploits the innovative technologies enabling the IoT in order to capture personal data for automatically recognizing behavioral changes in elderly people in an unobtrusive and low-cost manner. A specific module is introduced, which retrieves information about the interaction of the elderly with the surrounding environment.

In the work "Towards an adaptive SOA-based QoS \& Demand-Response Provisioning Architecture for the Smart Grid", [4] the authors C. Chrysoulas and M. Fasli present Service Oriented Architecture (SOA) for smart grid. Authors considered QoS attributes like: type of service to be served, response time, availability, and costs to be taken into consideration while sketching the proposed architecture in significant details.

In the work "Performance Evaluation of Routing Metrics in the LOADng Routing Protocol", [5] the authors J. V. V. Sobral, J. J. P. C. Rodrigues, N. Kumar, C. Zhu, R. Ahmad present evaluation of recently popular LOADng routing protocol as the alternative to RPL protocol widely used for IoT applications. Authors studied five different routing metrics (ETX, MBCR, MMBCR, LQI WL and MAXLQI) and the default routing metric of LOADng (HC) were studied in network scenarios with MP2P and P2P traffic patterns. The results obtained show that link based routing metrics were able to provide a high packet delivery ratio due to the use of most reliable paths. In contrast, these metrics have shown that, in some cases, the selection of reliable paths can use a high number of hop and provoke a power consumption increase.

In the work "An IC architecture for RF Energy Harvesting systems" [6], the authors L. Pantoli, A. Leoni, V. Stornelli, G. Ferri present novel hardware architecture energy harvester 
design for $900 \mathrm{MHz}$ band. Along with proposed architectuers, author's simulations ahow that the integrated system handles an incoming power typically ranging from $-45 \mathrm{dBm}$ to $5 \mathrm{dBm}$ by rectifying the variable input signals into a DC voltage source with an overall efficiency higher than $50 \%$.

In the work "An integrated access control and lighting configuration system for smart buildings", [7] the authors $\mathrm{H}$. S. Maciel, I. Cardoso, H. S. Ramos, J. J. P. C. Rodrigues, A. L. L. Aquino presented an integrated access control and lighting configuration system for smart buildings. The system uses two-factor authentication, one based on face recognition and other on RFID tag and identifies the user on the room and performs an automatic lighting configuration. The system can be easily deployed on meeting rooms or offices in business or government buildings. Evaluations show acceptable processing execution time, an acceptable communication time, by using a Radio Frequency solution, and the robustness of the system.

In the work "Ultra Wideband Assisted Localization of Semi-Autonomous Floor Scrubber", [8] the authors V. Celan, I. Stancic, J. Music presented a novel approach where standard floor-scrubber was upgraded with an additional both low cost and off-the shelf hardware and software platform.

In the paper "Design of Passive RFID Sensor Tags Enhanced by a Novel Logical Communication Procedure over LLRP" [9] the authors R. Colella, L. Catarinucci and L. Tarricone presented SPARTACUS, an enhanced version of passive RFID tag that exploits polarization diversity of two antennas performing both energy harvesting and communication, which ensures compactness, energyefficiency, and ease of use. The paper presents an implementation of a new physical layer that has been tested in some application contexts which take advantage from several features of the renovated device: the capability of asking for and receiving context data, sensing a physical value, reasoning and taking decisions.

Finally, in the paper "Electromagnetic Performance Estimation of UHF RFID Tags in Harsh Contexts" [10] the authors R. Colella, L. Catarinucci and L. Tarricone present a cost-effective and accurate tool for performance analysis of UHF passive RFID tags based on a commercial multiprogrammable UHF RFID reader controlling a stepper motor. Interestingly, the results obtained with commercial characterization platforms have demonstrated the validity of the proposed solution. The results obtained by characterizing different tags applied on, and embedded into concrete structures or placed around cylindrical structures, demonstrate the usefulness on the proposed system to be applied in real world scenarios in which the support in selecting or designing RFID tags is crucial.

\section{REFERENCES}

[1] L. Catarinucci, D. D. Donno, L. Mainetti, L. Patrono, M.L. Stefanizzi, L. Tarricone, "An IoT-aware Architecture to improve Safety in Sports Environments," Journal of Communication Software and Systems, vol. 12, no. 1, pp. XX, April, 2017.

[2] M. Zappatore, A. Longo and M. Bochicchio, "Crowd-sensing our Smart Cities: a Platform for Noise Monitoring and Acoustic Urban Planning," Journal of Communication Software and Systems, vol. 12, no. 1, pp. XX, April, 2016.
[3] L. Mainetti, L. Patrono, A. Secco and I. Sergi, "An IoT-aware AAL System to Capture Behavioral Changes of Elderly People," Journal of Communication Software and Systems, vol. 12, no. 1, pp. XX, April, 2016.

[4] C. Chrysoulas and M. Fasli, "Towards an adaptive SOA-based QoS \& Demand-Response Provisioning Architecture for the Smart Grid," Journal of Communication Software and Systems, vol. 12, no. 1, pp. XX, April, 2016.

[5] J. V. V. Sobral, J. J. P. C. Rodrigues, N. Kumar, C. Zhu and R. Ahmad, "Performance Evaluation of Routing Metrics in the LOADng Routing Protocol," Journal of Communication Software and Systems, vol. 12, no. 1, pp. XX, April, 2016.

[6] L. Pantoli, A. Leoni, V. Stornelli and G. Ferri, "An IC architecture for RF Energy Harvesting systems," Journal of Communication Software and Systems, vol. 12, no. 1, pp. XX, April, 2016.

[7] H. S. Maciel, I. Cardoso, H. S. Ramos, J. J. P. C. Rodrigues and A. L. L. Aquino, "An integrated access control and lighting configuration system for smart buildings," Journal of Communication Software and Systems, vol. 12, no. 1, pp. XX, April, 2016.

[8] V. Celan, I. Stancic, J. Music, "Ultra Wideband Assisted Localization of Semi-Autonomous Floor Scrubber," Journal of Communication Software and Systems, vol. 12, no. 1, pp. XX, April, 2016.

[9] R. Colella, L. Catarinucci and L. Tarricone, "Design of Passive RFID Sensor Tags Enhanced by a Novel Logical Communication Procedure over LLRP," Journal of Communication Software and Systems, vol. 12, no. 1, pp. XX, April, 2016.

[10] R. Colella, L. Catarinucci and L. Tarricone, "Electromagnetic Performance Estimation of UHF RFID Tags in Harsh Contexts," Journal of Communication Software and Systems, vol. 12, no. 1, pp. XX, April, 2016.

\section{GUEST EDITORS}

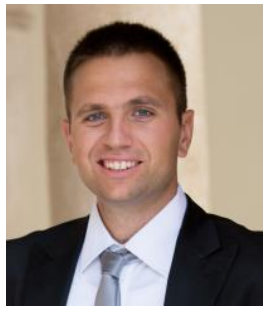

Petar Solic received his M.S. and Ph.D. degrees, both in computer science, from the University of Split in 2008 and 2014, respectively. He is currently employed at the Faculty of Electrical Engineering, Mechanical Engineering and Naval Architecture (FESB), University of Split, Croatia, as an assistant professor in the Department of Communication and Information Technologies. His research interests include information technologies, and RFID technology and its application. In 2016 he was awarded with National prize for science.

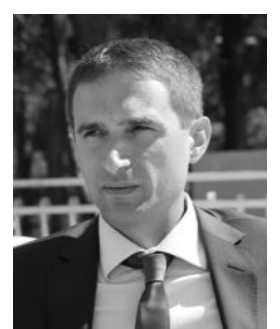

Luigi Patrono (luigi.patrono@unisalento.it) received his MS in Computer Engineering from University of Lecce, Lecce, Italy, in 1999 and $\mathrm{PhD}$ in Innovative Materials and Technologies for Satellite Networks from ISUFI-University of Lecce, Lecce, Italy, in 2003. $\mathrm{He}$ is an Assistant Professor of Computer Network at the University of Salento, Lecce, Italy. His research interests include RFID, EPCglobal, Internet of Things, Wireless Sensor Networks, and design and performance evaluation of protocols. He is Organizer Chair of the international Symposium on RFID Technologies and Internet of Things within the IEEE SoftCOM conference. He is Guest Editor of the Special Issue on RFID 
Technologies \& Internet of Things. He is author of about 100 scientific papers published on international journals and conferences and four chapters of books with international diffusion.

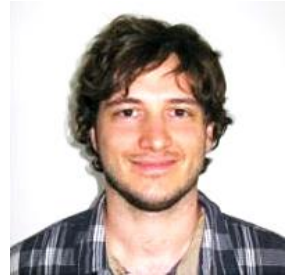

Toni Perković is currently employed as Assistant professor at University Department for Forensic Sciences, University of Split, Croatia. He received the Dipl. Ing. degree in telecommunications and electrical engineering from the University of Split, Croatia, in 2007, and the $\mathrm{PhD}$ degree in Computer Science from the University of Split, Croatia, in 2013. His research interests include the location privacy, security and privacy in Internet of Things, the usability, design and analysis of security protocols for wireless (sensor) networks, the usability and design of the secure authentication protocols.

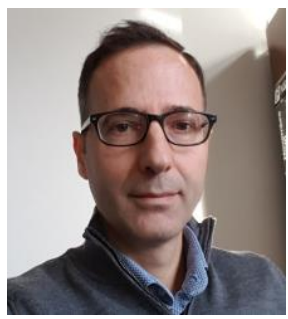

Luigi Atzori (SM’09) is Associate Professor at the Department of Electrical and Electronic Engineering at the University of Cagliari (Italy), where he leads the laboratory of Multimedia and Communications with around 15 affiliates (http://mclab.diee.unica.it). L. Atzori research interests are in multimedia communications and computer networking (wireless and wireline), with emphasis on multimedia QoE, multimedia streaming, NGN service management, service management in wireless sensor networks, architecture and services in the Internet of Things. He is the coordinator of the Marie Curie Initial Training Network on QoE for multimedia services (qoenet-itn.eu), which involves ten European Institutions in Europe and one in South Korea. He has been the editor for the ACM/Springer Wireless Networks Journal and guest editor for many journals, including the IEEE Communications Magazine, the Springer Monet and the Elsevier Signal Processing: Image Communications Journals. He is member of the steering committee for the IEEE Trans. on Multimedia, member of the editorial board of the IEEE IoT, the Elsevier Ad Hoc Networks and the Elsevier Digital Communications and Networks journals. He served as a technical program chair for various international conferences and workshops. He served as a reviewer and panelist for many funding agencies, including H2020, FP7, Cost and Italian MIUR. 